\title{
Prevalence of postoperative pressure ulcer: A systematic review and meta-analysis
}

\author{
Vida Shafipour ${ }^{1}$, Ensieh Ramezanpour ${ }^{2}$, Mohammad Ali Heidari Gorji ${ }^{1}$, Mahmood Moosazadeh $^{3}$
}

${ }^{1}$ Ph.D., Assistant Professor, Department of Medical-Surgical Nursing, Nasibeh Nursing \& Midwifery Faculty, Mazandaran University of Medical Sciences, Sari, Iran

${ }^{2}$ M.Sc. of Critical Care Nursing, Student's Research Committee, Nasibeh Nursing \& Midwifery Faculty Mazandaran University of Medical Sciences, Sari, Iran

${ }^{3}$ Ph.D. of Epidemiology, Assistant Professor, Health Science Research Center, Faculty of Health, Mazandaran University of Medical Sciences, Sari, Iran

Type of article: Meta-analysis

\begin{abstract}
Introduction: A pressure ulcer is a serious safety issue in healthcare systems. The patient's rate of infection with an ulcer, especially a postoperative ulcer, is critical, as it is dictated by factors such as being in a fixed position during surgery, the type of anesthesia used, the duration of surgery, and patient-related factors. The present study was conducted to carry out a systematic assessment of the prevalence of a postoperative pressure ulcer and to find its general prevalence using a meta-analysis.

Methods: The researchers searched databases, including PubMed, Google Scholar, Scopus, Science Direct, the Thomson Reuters' Web of Science (WOS). For English articles published online between January 2000 and October 2015 on the subject of a pressure ulcer, a total of 19 articles were ultimately selected based on the study inclusion criteria. Then results were analyzed in Stata-11.

Results: The 19 articles qualified for entering the meta-analysis examined a total of 9527 patients. The studies estimated the general prevalence of a postoperative pressure ulcer as 18.96\% (CI 95\%: 15.3-22.6); the prevalence by gender was reported as 10.1\% (CI 95\%: 7.2- 13.01) in men and $12.8 \%$ (CI 95\%: 8.3-17.2) in women. Stage 1 ulcer had a $17.02 \%$ prevalence (CI 95\%: 11.04- 22.9), stage 2 a $6.7 \%$ prevalence (CI 95\%: 3.8- 9.7), stage 3 a $0.9 \%$ prevalence (CI 95\%: 0.2-1.6), and stage 4 a $0.4 \%$ (CI 95\%: -0.05-0.8) prevalence.

Conclusion: The prevalence of a postoperative pressure ulcer is high among the entire population; however, it is still higher in women than in men. The prevalence of a stage 1 ulcer is higher than the prevalence of the other stages of an ulcer.
\end{abstract}

Keywords: Pressure ulcer, Prevalence, Risk factors, Surgical patients

\section{Introduction}

\subsection{Background}

A pressure ulcer is a serious safety issue in healthcare systems (1) and adversely affects patients due to the pain it causes and the time it takes to heal. These ulcers also increase the workload of health care professionals and, as a consequence, increase health care costs dramatically. In extreme situations, pressure ulcers can cause death. National programs have been initiated to manage this problem and to stimulate preventive actions (2). The National Pressure Ulcer Advisory Panel (NPUAP) defines a pressure ulcer as the impaired integrity of the skin caused by the compression of the soft skin tissue between the bony processes and an external surface. This ulcer is caused by an impaired tissue perfusion and a subsequent partial loss of the body cells progressively destroying the underlying layers $(3,4)$. The duration of compression that the skin tolerates until it breaks down varies from one patient to another; in many incapable patients, tissue damage occurs within less than two hours (5). In terms of severity, the NPUAP has defined four stages for a pressure ulcer. A stage 1 pressure ulcer consists of a reddened spot that does

\section{Corresponding author:}

Assistant Professor Dr. Mahmood Moosazadeh, Health Science Research Center, Faculty of Health, Mazandaran University of Medical Sciences, Sari, Iran. Tel:+98.9113555367, Email: mmoosazadeh1351@gmail.com Received: April 06, 2016, Accepted: July 26, 2016, Published: November 2016 iThenticate screening: July 26, 2016, English editing: September 10, 2016, Quality control: October 23, 2016 (C) 2016 The Authors. This is an open access article under the terms of the Creative Commons Attribution-NonCommercialNoDerivs License, which permits use and distribution in any medium, provided the original work is properly cited, the use is non-commercial and no modifications or adaptations are made. 
not blanch by pressing and may be accompanied with edema. A stage 2 pressure ulcer consists of damage to the surface layers of the skin, i.e., the epidermis or the dermis or even both; a stage 2 ulcer is often superficial and presents as a painful scratch, blister, or small cavity. In a stage 3 ulcer, the skin thickness is completely lost, and the subcutaneous tissue damage extends deep into the muscle tissue. At this point, the ulcer is clinically observed as a deep cavity that might extend to the surrounding tissues and take months to heal. A stage 4 ulcer involves the complete loss of the skin together with an extensive skin destruction and necrosis and the involvement of the muscles, bones, and tendons. Secretory sinuses also may occur at this stage (5). This classification involves an unclassified stage as well, in which the tissue thickness is completely lost, and the ulcer is covered with a yellow, gray, green, or brown tissue, which has to be removed for the depth and stage of the ulcer to be determined $(3,6)$. Study question: What is the prevalence of a postoperative pressure ulcer by gender and ulcer stages?

\subsection{Statement of the Problem}

The rate of infection with an ulcer, especially a postoperative ulcer, is important due to factors such as being in a fixed position during surgery, the method of anesthesia used, the duration of surgery, and patient-related factors such as age, gender, underlying diseases (diabetes, heart failure, etc.). A postoperative pressure ulcer is one of the causes of prolonged hospital stays and increased costs and workloads within the healthcare system (7). The treatment of a pressure ulcer costs between $\$ 2000$ and $\$ 70,000$ per patient according to the intensity and stage of the ulcer, and the annual cost of treating pressure ulcer incurred by hospitals is between $\$ 400,000$ and $\$ 700,000$ (8). In the United States, approximately 1.6 billion patients develop health care associated pressure ulcers at an annual cost of $\$ 2.2$ billion to $\$ 3.6$ billion (9).

\subsection{Objective of Research}

Implementing preventive measures therefore requires the identification of patients at risk for developing a pressure ulcer and the investigation of its postoperative prevalence. The initial search carried out in this study revealed the prevalence of a pressure ulcer to range from $8.1 \%$ to $27.3 \%$ in the United States $(10,11)$ and from $11.1 \%$ to $54.8 \%$ in Turkey $(12,13)$ and to be $21.3 \%$ in Iran $(14)$. Given the substantial difference in the prevalence rates reported for pressure ulcers in different studies, the present study seeks to integrate the results obtained from the initial studies examined through a meta-analysis and a systematic review and to present credible evidence on the subject. This study thus examines the prevalence of a postoperative pressure ulcer in general and by gender and ulcer stage.

\section{Material and Methods \\ 2.1. Study type}

The present study was conducted to investigate the prevalence of a postoperative pressure ulcer in general and by gender and ulcer stage using a systematic review and a meta-analysis.

\subsection{Search Strategy}

To access English articles published online between January 2000 and October 2015 on the subject of pressure ulcers, databases including the PubMed, Google Scholar, Scopus, Science Direct, and the Thomson Reuters' Web of Science (WOS) were searched. The search was carried out between August 23 and October 23, 2015, of article abstracts and titles using a combination of major English keywords on the subject, including pressure ulcer, prevalence, risk factors, and surgical patients. To increase the sensitivity of the search and enable a greater choice of articles that could not be retrieved through the databases, the list of references inserted in these articles was also examined. The retrieved articles were evaluated by two of the researchers at random, and no articles were excluded.

\subsection{Article selection}

The full text or the abstract of all the articles, documents, and reports yielded by the advanced search were extracted, and repeated items were excluded. Irrelevant articles also were excluded with a review of the title, abstract, and full text of the articles as well as the authors' names, publication year, and journal number and title. To prevent reprint bias (transverse and longitudinal bias), the results were examined by the researchers to identify and eliminate repeated articles. All the articles written in English and examining the prevalence of postoperative pressure ulcers in adults entered the systematic review and meta-analysis once they were evaluated and reported to meet the minimum requirements. The articles that did not report the prevalence of a pressure ulcer or were conducted on children, control-case or interventional studies, or studies that did not receive the required score according to the checklist were excluded from the study. 


\subsection{Quality assessment}

After identifying the articles that were relevant in terms of title and content, their quality was assessed using a checklist used in previous studies (15) and developed according to the strengthening the reporting of observational studies in an epidemiology (STROBE) checklist. The checklist consists of 12 questions that examine different methodological aspects of a study, including appropriate sample size, type of study, sampling method, study population, data collection method, variable definition, sample assessment method, data collection tools, statistical tests employed, study objectives, and the appropriate presentation of the results in accordance with the study objectives. One point is allocated to each question and any article receiving a score of 12 thus entered the metaanalysis (15).

\subsection{Data extraction}

The data pertaining to each article was extracted by title, the corresponding author's name, study year, study setting, sample size, sample size by gender, the prevalence of pressure ulcer in general and by gender and ulcer stage, study type, sampling method, and study method. The data were entered into an Excel spreadsheet by two of the researchers.

\subsection{Analysis}

Data were analyzed in Stata. The binomial distribution formula was used to calculate the standard error in the prevalence of pressure ulcer reported in each article. The heterogeneity index was then determined using Cochran's Q test. Based on the results obtained, the randomized effect model was used in the meta-analysis using the meta command for estimating the prevalence of a postoperative pressure ulcer. In addition, to minimize the randomized distribution of the point estimates of the articles, the data obtained from all the articles were adjusted using Bayesian analysis. The meta-regression command was then used in Stata-11 to examine the effects of the variables suspected as potential sources of heterogeneity. The point estimate of the prevalence of a pressure ulcer was calculated in forest plots with a $95 \%$ confidence interval; in this plot, the size of the square indicates the weight of each article, and the lines on the sides show a $95 \%$ confidence interval.

\section{Results}

The initial search in international databases led to the identification of 103 articles. Imposing further constraints and increasing the specificity of the search then reduced the number of nearly relevant articles to 90,30 of which were repetitive due to the overlap between databases. The titles and abstracts of the remaining 60 articles were reviewed, and 15 were excluded due to being irrelevant to the objectives of the review study. The review of the full text of the remaining 45 articles led to the selection of 30,15 of which ultimately entered the study after they were fully assessed in terms of meeting the inclusion and exclusion criteria and the minimum quality requirements. The article references also were reviewed and thus led to the identification of four additional articles relevant to the subject. A total of 19 articles ultimately entered the meta-analysis and systematic review stages of the study (Figure 1). All 19 articles selected were descriptive, analytical and cross-sectional and reported the general prevalence of postoperative pressure ulcer. Eight articles reported this prevalence in men and eight reported it in women. Ten articles reported the prevalence of stage 1 ulcer, 11 the prevalence of stage 2 ulcer, five the prevalence of stage 3 ulcer, and four the prevalence of stage 4 ulcer (Table 1).

\subsection{General prevalence of postoperative pressure ulcer}

All 19 articles (Figure 2) reported the general prevalence of postoperative pressure ulcer and examined a total of 9527 patients. The general prevalence of postoperative pressure ulcer varied from $5.1 \%$ in the study by Shaw (6) with a sample size of 297 to $64.1 \%$ in the study by Feuchtinger (17) with a sample size of 53 (Table 1). Due to the presence of heterogeneity, the randomized effect model was used to report the general prevalence of the ulcer, which is estimated as $18.96 \%(15.3,22.6)$ (Table 2$)$.

\subsection{Prevalence of postoperative pressure ulcer by gender}

The minimum prevalence of pressure ulcer was reported as $4.5 \%$ in men in the study by Pokorny et al. conducted on a sample of 222 men (18), and its maximum was reported as $18.5 \%$ in the study by Lumbley et al., which was conducted on a sample of 553 men (10). In the present study, the review of the eight articles that examined a total of 3632 men showed the prevalence of an ulcer to be $10.1 \%(7.2,13.01)$ in men. The minimum prevalence of postoperative pressure ulcer was reported as $4.8 \%$ in women in a study by Primiano et al. conducted on a sample of 147 women (9), and its maximum was reported as $40 \%$ in a study by Shadedi et al. conducted on a sample of 100 
women (19). In the present study, the review of the eight articles that examined a total of 2540 women showed the prevalence of ulcer to be $12.8 \%$ (CI 95\%: 8.3-17.2) in women (Table 2).

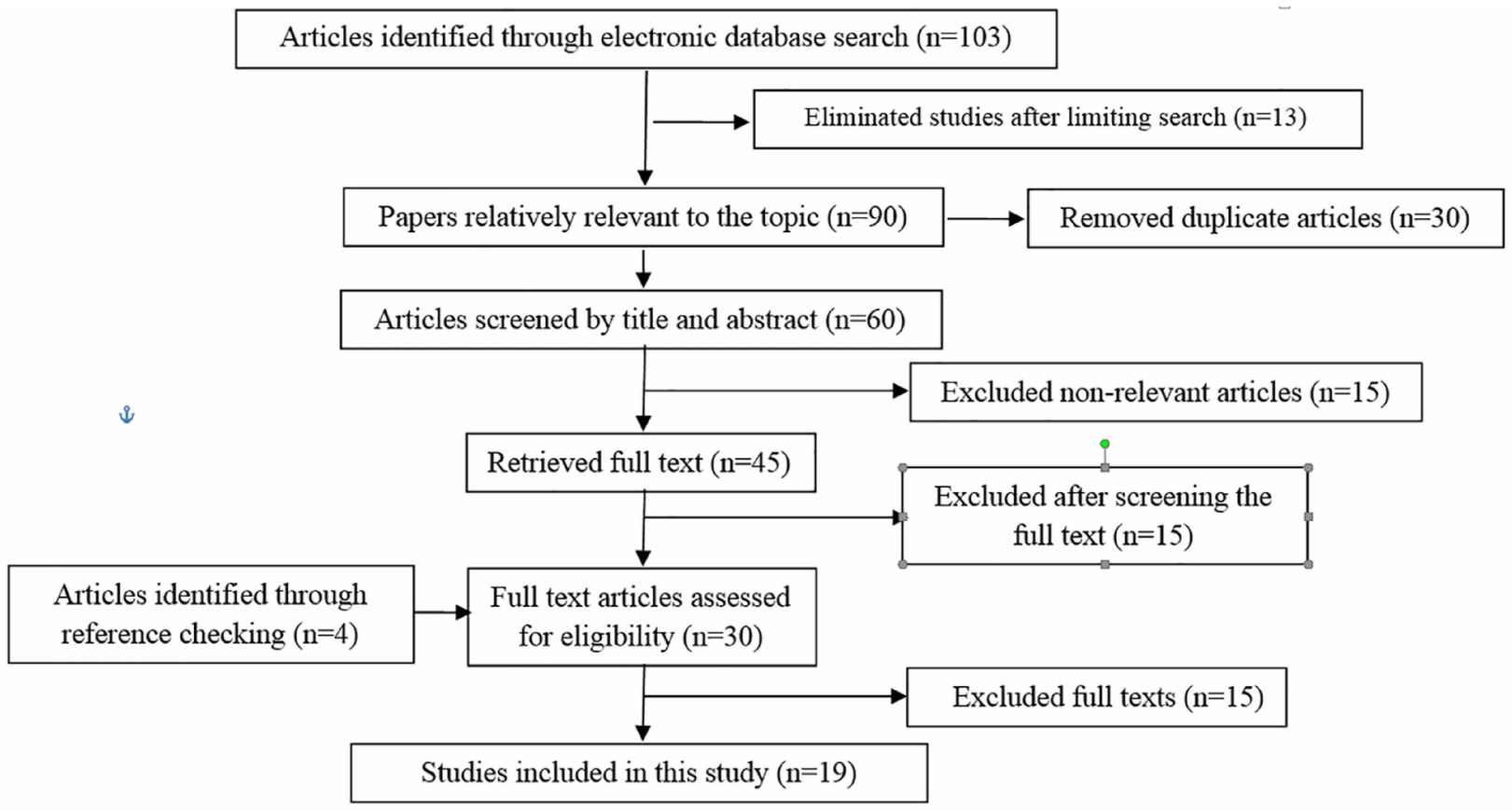

Figure 1. Literature search and review flow chart for selection of primary studies.

Table 1. Prevalence of pressure ulcer (\%) by articles entering the meta-analysis

\begin{tabular}{|l|l|l|l|l|l|l|l|l|l|}
\hline \multirow{2}{*}{ First author } & $n$ & Total $\mathrm{P}^{3}$ & $\mathrm{MP}^{4}$ & $\mathrm{FP}^{5}$ & $\mathrm{p}$-stage 1 & p-stage2 & p-stage 3 & p-stage 4 \\
\cline { 2 - 11 } & $\mathrm{M}^{1}$ & $\mathrm{~F}^{2}$ & & & & & & & \\
\hline Schoonhoven & 136 & 72 & 21.1 & & & 11 & 10.1 & & \\
\hline Pokorny & 222 & 129 & 7 & 4.5 & 10.8 & 4.3 & 1.99 & 0.28 & 0.28 \\
\hline Lindgren & 157 & 129 & 14.3 & 7.6 & 22.5 & 9.79 & 3.5 & 1 & \\
\hline Nixon & 38 & 59 & 15.5 & & & & 13.4 & & 1.7 \\
\hline Karadag & & & 54.8 & & & 54.8 & & & \\
\hline Sewchuk & 100 & 50 & 11.3 & & & 3.33 & 5.3 & 2 & 1.3 \\
\hline Feuchtinger & 31 & 22 & 64.1 & & & 62.2 & 1.8 & & \\
\hline Rademarkers & 171 & 551 & 30 & & & & 27.5 & 2.07 & \\
\hline Scarlatti & 101 & 98 & 20.6 & & & 13 & 7 & 0.28 & 0.28 \\
\hline KAMpbell & 28 & 44 & 17 & & & 3 & 7 & & \\
\hline Tschanen & 1681 & 1161 & 12 & 13.6 & 13.2 & & & & \\
\hline Shen & & & 16.4 & & & 16.08 & 0.35 & & \\
\hline Primiano & 108 & 147 & 8.1 & 12.03 & 4.8 & & & & \\
\hline Esoppi & 1025 & 604 & 11.1 & 12.2 & 9.3 & & & & \\
\hline Lumbley & & & 27.3 & 18.5 & 8.7 & & & & \\
\hline Shadedi & 115 & 100 & 27.9 & 17.3 & 40 & & & & \\
\hline Honglinchen & 160 & 126 & 16.4 & & & 16 & 0.35 & & \\
\hline Shaw & 162 & 135 & 9.8 & 9.8 & 9.6 & & & & \\
\hline Shaw & 162 & 135 & 5.1 & 6.1 & 3.7 & & & & \\
\hline
\end{tabular}

1: male; 2: female; 3 : total prevalence; 4: male prevalence; 5 : female prevalence 


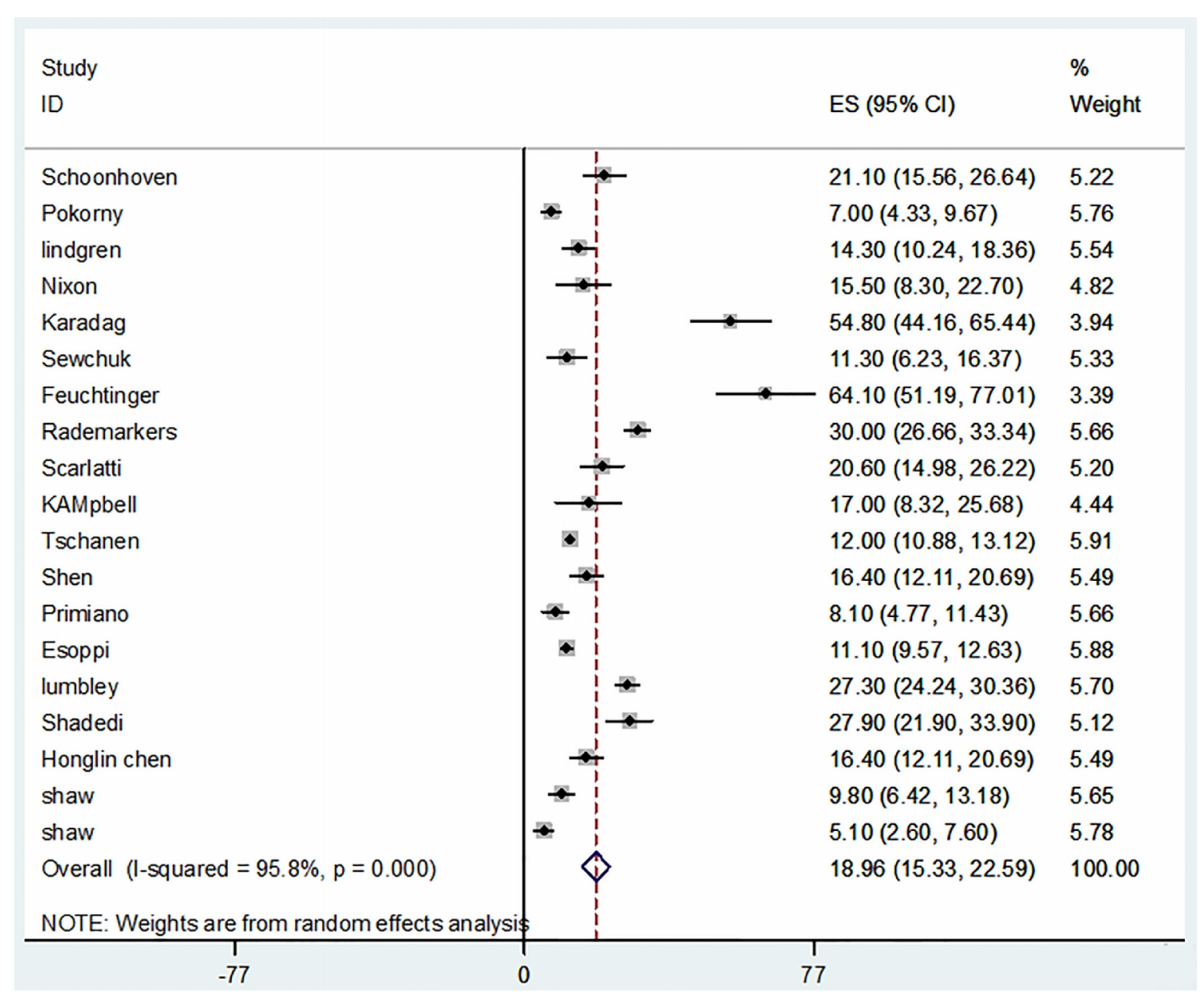

Figure 2. General prevalence of postoperative pressure ulcer in each article and the overall estimate.

Table 2. Prevalence of postoperative pressure ulcer based on the meta-analysis

\begin{tabular}{|l|l|l|l|l|l|l|l|}
\hline Prevalence & Number of primary studies & Sample size & Prevalence & CI 95\% & \multicolumn{3}{|l|}{ Heterogeneity } \\
\cline { 5 - 8 } & & & & & Q & I-squared & p-value \\
\hline Total & 19 & 9527 & 18.96 & $15.3-22.6$ & 425.9 & 95.8 & $<0.001$ \\
\hline Male & 8 & 3632 & 10.1 & $7.2-13.01$ & 45.8 & 84.7 & $<0.001$ \\
\hline Female & 8 & 2540 & 12.8 & $8.3-17.2$ & 83.3 & 91.6 & $<0.001$ \\
\hline Stage 1 & 10 & 1975 & 17.02 & $11.04-22.9$ & 202.2 & 95.5 & $<0.001$ \\
\hline Stage 2 & 11 & 2710 & 6.7 & $3.8-9.7$ & 316.02 & 96.8 & $<0.001$ \\
\hline Stage 3 & 5 & 1708 & 0.9 & $0.2-1.6$ & 11.6 & 65.6 & 0.02 \\
\hline Stage 4 & 4 & 797 & 0.4 & $-0.05-0.8$ & 2.2 & 0 & 0.5 \\
\hline
\end{tabular}

\subsection{Prevalence of postoperative pressure ulcer by stage of ulcer}

Ten articles examined the prevalence of a stage 1 ulcer in a sample of 1975 patients and reported it as $17.02 \%$ (CI 95\%: 11.04- 22.9); 11 articles examined the prevalence of a stage 2 ulcer in a sample of 2710 patients and reported it as 6.7\% (CI 95\%: 3.8-9.7); five articles examined the prevalence of a stage 3 ulcer in a sample of 1708 patients and reported it as $0.9 \%$ (CI 95\%: 0.2-1.6 and four articles examined the prevalence of a stage 4 ulcer in a sample of 797 patients and reported it as $0.4 \%$ (CI 95\%: -0.05-0.8) (Table 2).

\subsection{Risk factors associated with the pressure ulcers}

The present study also examined some of the preoperative factors associated with the development of a pressure ulcer, including age, gender, BMI, and underlying diseases such as heart failure and diabetes. Age was found to be a significant factor in four of the reviewed studies $(11,18,20)$, gender in two $(18,20)$, BMI in two $(11,20)$, serum albumin levels in one (20), and each underlying disease also in one (heart failure (18), musculoskeletal disorders 
(20), diabetes (11), and smoking (16). Other factors also were each found in one article to contribute significantly to the development of pressure ulcer, including the number of surgeries since admission (11), the duration of hospitalization until the surgical operation (18), the risk of mortality (11), and the preoperative Braden Score (11). Some operative factors also were found to contribute significantly to the development of a pressure ulcer, including the body position during surgery (21), the duration of surgery $(11,21,22)$, and the type of anesthesia used (20). The postoperative factors that contributed significantly to the development of pressure ulcer included the postoperative Braden Score (18), postoperative urinary tract infection (17), and prolonged hospital stay after the surgery (17).

\section{Discussion}

Meta-analysis of the studies conducted on a total 9527 surgery patients showed the prevalence of postoperative pressure ulcer to be $18.96 \%$. The prevalence of a pressure ulcer was reported as $12.8 \%$ in women according to the sample of 2540 women examined, and as $10.1 \%$ in men according to the sample of 3632 men examined. Despite the larger number of men who had developed this ulcer, the prevalence of a pressure ulcer was deemed to be higher in women than in men, which is consistent with the results of some studies $(18,20)$ and inconsistent with the results of others $(9,11,16)$, which reported the prevalence to be higher in men. In the present study, the prevalence of a stage 1 ulcer was $17.02 \%$, which is higher than the prevalence of the other stages of an ulcer. This finding is consistent with the results of some studies $(12,22,23)$ and inconsistent with the results of others $(24-26)$, which reported the prevalence of a stage 2 ulcer to be higher. The personal risk factors associated with the development of postoperative pressure ulcer included age, gender, BMI, underlying diseases such as diabetes, heart failure, musculoskeletal disorders, and smoking, as well as the number of surgeries since admission (11), the duration of hospitalization until the time of surgical operation (19), the risk of mortality (11), and the preoperative Braden Score (11). The operative factors affecting the development of an ulcer included the body position during surgery (11), the duration of surgery $(11,22,23)$, and the type of anesthesia used (24); the postoperative factors contributing to the development of an ulcer included the postoperative Braden Score (18), postoperative urinary tract infection (17) and prolonged hospital stay after the surgery (17). According to the results obtained, women are more prone to developing postoperative pressure ulcer than men, with older patients and those with underlying diseases such as diabetes, heart failure, and musculoskeletal disorders, and lower Braden Scores also being at a greater risk for developing this ulcer. Patients with a prolonged duration of surgery and those experiencing postoperative urinary tract infection also are at a greater risk of developing pressure ulcer.

\section{Conclusions}

Our studies show that prevalence of a postoperative pressure ulcer is $18.96 \%$, and patients with a prolonged duration of surgery and those experiencing postoperative urinary tract infection also are at a greater risk of developing a pressure ulcer. The results of the present study can be used by policymakers for improving prevention programs implemented before, during, and after surgery, on the one hand, and, on the other, for identifying patients at a greater risk of developing an ulcer, so as to help prevent its incidence and reduce hospital stays and treatment costs.

\section{Acknowledgments:}

We hereby thank all the people who contributed to this research.

\section{Conflict of Interest:}

There is no conflict of interest to be declared.

Authors' contributions:

All authors contributed to this project and article equally. All authors read and approved the final manuscript.

\section{References:}

1) Baranoski S, Ayello EA. Wound care essentials: Practice principles. 2008: Lippincott Williams \& Wilkins.

2) Girouard K, Harrison MB, VanDenKerkof E. The symptom of pain with pressure ulcers: a review of the literature. Ostomy Wound Manage. 2008; 54(5): 30-40, 42. PMID: 18493092.

3) Perry AG, Potter PA, Ostendorf. Clinical Nursing Skills and Techniques, 8th Edition. Elsevier Health Sciences. 2013.

4) Black JM, Hawks JH. Medical-Surgical Nursing: Clinical Management for Positive Outcomes (Single Volume), 8th Edition. 2001; 1.

5) Black J, Baharestani MM, Cuddigan J, Dorner B, Edsberg L, Langemo D, et al. National Pressure Ulcer Advisory Panel's updated pressure ulcer staging system. Adv Skin Wound Care. 2007; 20(5): 269-74. doi: 10.1097/01.ASW.0000269314.23015.e9. PMID: 17473563. 
6) Thomas DR. Prevention and treatment of pressure ulcers: what works? what doesn't? Cleve Clin J Med. 2001; 68(8): 704-7, 710-14, 717-22. doi: 10.3949/ccjm.68.8.704. PMID: 11510528.

7) Palfreyman SJ, Stone PW. A systematic review of economic evaluations assessing interventions aimed at preventing or treating pressure ulcers. Int J Nurs Stud. 2015; 52(3): 769-88. doi: 10.1016/j.ijnurstu.2014.06.004. PMID: 25012958.

8) Carson D, Emmons K, Falone W, Preston AM. Development of pressure ulcer program across a university health system. J Nurs Care Qual. 2012. 27(1): 20-7. doi: 10.1097/NCQ.0b013e3182310f8b. PMID: 21915063.

9) Primiano M, Friend M, McClure C, Nardi S, Fix L, Schafer M, et al. Pressure ulcer prevalence and risk factors during prolonged surgical procedures. AORN J. 2011; 94(6): 555-66. doi: 10.1016/j.aorn.2011.03.014. PMID: 22118201, PMCID: PMC4467017.

10) Lumbley JL, Ali SA, Tchokouani LS. Retrospective review of predisposing factors for intraoperative pressure ulcer development. J Clin Anesth. 2014; 26(5): 368-74. doi: 10.1016/j.jclinane.2014.01.012. PMID: 25113424.

11) Tschannen D, Bates O, Talsma A, Guo Y. Patient-specific and surgical characteristics in the development of pressure ulcers. Am J Crit Care. 2012; 21(2): 116-25. doi: 10.4037/ajcc2012716. PMID: 22381988.

12) Karadag M, Gümüskaya N. The incidence of pressure ulcers in surgical patients: a sample hospital in Turkey. J Clin Nurs. 2006; 15(4): 413-21. doi: 10.1111/j.1365-2702.2006.01369.x. PMID: 16553754.

13) Ahtiala MH, Soppi ET, Wiksten A, Koskela H, Grönlund JA. Occurrence of Pressure Ulcers and Risk Factors in a Mixed Medical-Surgical ICU-A Cohort Study. Journal of the Intensive Care Society. 2014; 15(4): 340-3. doi: 10.1177/175114371401500415.

14) Alizadeh Ghavidel A, Bashavard S, Abkenar B, Payghambari MM. Incidence rate of pressure sores after cardiac surgery during hospitalization and its relevant factors. Razi Journal of Medical Sciences. 2012; 19(102): 18-29.

15) Moosazadeh M, Nekoei-Moghadam M, Emrani Z, Amiresmaili M. Prevalence of unwanted pregnancy in Iran: a systematic review and meta - analysis. Int J Health Plann Manage. 2014; 29(3): 277-90. doi: 10.1002/hpm.2184. PMID: 23630092.

16) Shaw LF, Chang PC, Lee JF, Kung HY, Tung TH. Incidence and predicted risk factors of pressure ulcers in surgical patients: experience at a medical center in Taipei, Taiwan. Biomed Res Int. 2014; 2014: 416896. doi: 10.1155/2014/416896. PMID: 25057484, PMCID: PMC4099038.

17) Feuchtinger J, Halfens R, Dassen T. Pressure ulcer risk assessment immediately after cardiac surgery--does it make a difference? A comparison of three pressure ulcer risk assessment instruments within a cardiac surgery population. Nurs Crit Care. 2007; 12(1): 42-9. doi: 10.1111/j.1478-5153.2006.00198.x. PMID: 17883663.

18) Pokorny ME, Koldjeski D, Swanson M. Skin care intervention for patients having cardiac surgery. Am J Crit Care. 2003. 12(6): 535-44. PMID: 14619359.

19) Shadedi AL. Prevalence of pressure ulcers in orthopedic patients. 2014.

20) Lindgren M, Unosson M, Krantz AM, Ek AC. pressure ulcer risk factors in patients under going surgery. J Adv Nurs. 2005; 50(6): 605-12. doi: 10.1111/j.1365-2648.2005.03441.x. PMID: 15926965.

21) Scarlatti KC, Michel JL, Gamba MA, de Gutiérrez MG. [Pressure ulcers in surgery patients: incidence and associated factors]. Rev Esc Enferm USP. 2011; 45(6): 1372-9. doi: 10.1590/S0080-62342011000600014. PMID: 22241195.

22) Shen WQ, Chen HL, Xu YH, Zhang Q, Wu J. The Relationship Between Length of Surgery and the Incidence of Pressure Ulcers in Cardiovascular Surgical Patients: A Retrospective Study. Adv Skin Wound Care. 2015; 28(10): 444-50. doi: 10.1097/01.ASW.0000466365.90534.b0. PMID: 26375947.

23) Schoonhoven L, Defloor T, van der Tweel I, Buskens E, Grypdonck MH. Risk indicators for pressure ulcers during surgery. Appl Nurs Res. 2002; 15(3): 163-73. PMID: 12173167.

24) Nixon J, Cranny G, Bond S. Skin alterations of intact skin and risk factors associated with pressure ulcer development in surgical patients: a cohort study. Int J Nurs Stud. 2007; 44(5): 655-63. doi: 10.1016/j.ijnurstu.2006.02.010. PMID: 16631178.

25) Campbell KE, Woodbury G, Labate T, LeMesurier A, Houghton PE. Heel ulcer incidence following orthopedic surgery: a prospective, observational study. Ostomy Wound Manage. 2010; 56(8): 32-9. PMID: 20729561.

26) Rademakers LM, Vainas T, van Zutphen SW, Brink PR, van Helden SH. Pressure Ulcers and Prolonged Hospital Stay in Hip Fracture Patients Affected by Time-to-Surgery. Eur J Trauma Emerg Surg. 2007; 33(3): 238-44. doi: 10.1007/s00068-007-6212-8. PMID: 26814486. 